\title{
Clinical Characteristics of 18 Patients with Psoriasis and Multiple Myeloma Identified Through Digital Health Crowdsourcing
}

\author{
Joy Q. Jin (D) · Jenny M. Ahlstrom - Nathan W. Sweeney • \\ Wilson Liao (D)
}

Received: May 20, 2020 / Published online: July 7, 2020

(C) The Author(s) 2020

\begin{abstract}
Psoriasis is a skin condition that affects over 100 million people worldwide, while multiple myeloma (MM) accounts for $10 \%$ of all hematologic malignancies in the US. There has been limited research on the intersection of psoriasis and $\mathrm{MM}$, and clinicians often face difficult decisions in treating patients diagnosed with both conditions. For instance, the management of psoriasis with systemic immunotherapies in MM patients can be challenging because of concern about immunosuppression and possible worsening of MM. Online crowdsourcing platforms have recently become innovative tools that can actively empower patients in
\end{abstract}

Digital Features To view digital features for this article go to https://doi.org/10.6084/m9.figshare.12514808.

Electronic supplementary material The online version of this article (https://doi.org/10.1007/s13555020-00416-5) contains supplementary material, which is available to authorized users.

\section{J. Q. Jin}

School of Medicine, University of California San

Francisco, San Francisco, USA

J. M. Ahlstrom · N. W. Sweeney

Myeloma Crowd, CrowdCare Foundation, Lehi, UT, USA

\section{W. Liao $(\bowtie)$}

Department of Dermatology, University of

California San Francisco, San Francisco, USA

e-mail: Wilson.Liao@ucsf.edu scientific research by enabling the contribution of health data. One such platform, HealthTree ${ }^{\circledR}$, helps MM patients find optimal myeloma treatments and has registered $>6000$ patients, many of whom have uploaded medical records and genetic profiles. By taking advantage of patient health data available on HealthTree, researchers can gain a greater understanding of the clinical characteristics and treatment responses of patients diagnosed with psoriasis and MM. In this case series, we first report a psoriasis and MM patient treated with the IL-17 inhibitor ixekizumab who demonstrated a temporary, 2-month improvement in $\mathrm{MM}$ biomarkers (M-protein, kappa, and kappa:lambda ratio). We then report on the clinical characteristics of 18 patients with verified profiles on HealthTree indicating concurrent psoriasis and MM conditions. We surveyed gender, age, psoriasis type, psoriasis treatment history, myeloma type, myeloma genetic features, and myeloma association with bone damage, hypercalcemia, or osteopenia. Four patients were treated with systemic immunomodulators for psoriasis, with responses suggesting that these therapies did not worsen MM progression. Our results validate crowdsourcing as a way to assess patient demographics and treatment responses for use in dermatology research. We examine the demographics of patients diagnosed with psoriasis and $\mathrm{MM}$ and investigate the use of systemic immunomodulators for treatment of psoriasis in MM patients. 
Keywords: Crowdsource; HealthTree; IL-17; Ixekizumab; Multiple myeloma; Patient empowerment; Psoriasis; Systemic therapy

\section{Key Summary Points}

\section{Why carry out this study?}

There has been limited research on the intersection of psoriasis and multiple myeloma (MM), and providers often face difficult decisions in treating patients diagnosed with both conditions

Interleukin-17 (IL-17) has been identified as a cytokine that promotes both psoriasis and MM; however, the use of IL-17 inhibitors in patients with MM has not been examined

By crowdsourcing patient health data using the HealthTree platform, we can gain a greater understanding of the clinical characteristics and treatment responses of patients diagnosed with both psoriasis and $\mathrm{MM}$

\section{What was learned from the study?}

We identified a patient with psoriasis and active MM who was treated with the IL-17 inhibitor ixekizumab and who then experienced a transient reduction in her MM serologic markers

To further study psoriasis and MM, we identified 18 patients on HealthTree, a MM crowdsourcing platform. We analyzed information on gender, age, psoriasis type and treatment history, myeloma type and genetic features, and myeloma association with bone damage, hypercalcemia, or osteopenia

Among the 18 subjects, 4 patients were treated with a systemic immunomodulator for psoriasis with no obvious worsening of MM progression

\section{INTRODUCTION}

Traditionally, patients have been difficult to engage in clinical research and analysis. However, online crowdsourcing platforms have become innovative tools that can empower and actively engage patients in the research process by enabling the contribution of health data, ideas, and observations. Platforms such as CureTogether and Citizen Pscientist have enrolled thousands of patients to generate deidentified patient data for clinical study [1]. The recently launched HealthTree ${ }^{\circledR}$ platform, an online ecosystem that helps multiple myeloma patients find their best myeloma treatments and accelerate research toward a cure, has registered $>6000$ patients, many of whom have uploaded verified medical records and genetic profiles [2].

To date, there has been limited research on the intersection between psoriasis and multiple myeloma (MM), and providers often face difficult decisions in treating patients diagnosed with both conditions. Psoriasis is a chronic inflammatory skin disease that is estimated to affect $>100$ million people worldwide [3]. MM accounts for $10 \%$ of all hematologic malignancies, with $>30,000$ new cases diagnosed in the US each year [4]. Although there are many effective systemic treatments for psoriasis, including therapies targeting tumor necrosis factor $\alpha$ (TNF- $\alpha$ ), interleukin 17 (IL-17), and interleukin 23 (IL-23), the use of systemic agents in patients with malignancies is controversial because of concerns of immunosuppression and potential cancer progression. This is particularly true for hematologic malignancies, in which elevated rates of lymphoma in patients receiving anti-TNF agents have been reported [5].

Here, we present a case series analyzing the deidentified health data of 18 patients enrolled in HealthTree who were diagnosed with psoriasis and MM. The study results indicate that medical research can be greatly enhanced by crowdsourcing patient health data via online platforms, especially for intersecting patient populations such as those diagnosed with both $\mathrm{MM}$ and psoriasis. Our findings provide further insight for the management of systemic 
psoriatic therapies in patients with existing myeloma. Consent for publication was received from the index patient; the digitally crowdsourced patient data were determined to be exempt by the Western Institutional Review Board (IRB) under exemption code 45 CFR § 46.104(d)(2). This case report was conducted according to the principles of good clinical practice guidelines ICH GCP and according to the ethical principles of the Declaration of Helsinki.

\section{CASE PRESENTATIONS}

\section{Index Case}

We first evaluated Patient 1, a 52-year-old female with a 30-year history of guttate psoriasis without psoriatic arthritis. Her psoriasis was generally well managed with topical therapy (clobetasol and hydrocortisone creams) and heliotherapy.

At age 43, Patient 1 was diagnosed with Durie-Salmon stage 3A IgG kappa MM, with genetic profiling showing trisomy 9 and 11, tetrasomy 15 , and a $t(14 ; 20)$ translocation. She was found to have diffuse osteolytic lesions throughout the body, and subsequently underwent dexamethasone, cisplatin, doxorubicin, cyclophosphamide and etoposide (D-PACE) and bortezomib, thalidomide, and dexamethasone (VTD) chemotherapy treatment. Tandem autologous stem cell transplantation (ASCT) was performed, followed by a year of maintenance therapy with VTD and an additional year with dexamethasone, after which Patient 1 achieved a complete remission (CR) approximately 1 year after initial MM diagnosis. Dexamethasone maintenance was started immediately for 1 year, and the patient remained in stringent $\mathrm{CR}$ with consistent monitoring.

However, at age 48, despite no evidence of active myeloma on imaging, bone marrow biopsy showed $5 \%$ presence of plasma cells, which is at the borderline level for MM recurrence. Osteoporosis-preventing infusions were subsequently administered every 3 months and markers were closely tracked for the following
4 years; M-protein and serum markers (e.g., albumin, creatinine) remained stable, and no acute findings or evidence of active disease progression was noted.

At age 52, Patient 1 was noted to have a sharp increase in $\mathrm{MM}$ biomarkers, including kappa and urine paraprotein. She also developed a psoriatic flare with lesions noted on the posterior neck, right lower abdomen, and chest. There was mild scaling and induration of psoriatic lesions, and she was prescribed topical creams and started on ixekizumab therapy. Ixekizumab was selected based on its established efficacy in psoriasis and studies suggesting a pathogenic role for IL-17 in the progression of MM [6]. Twelve days after the first loading dose injection of ixekizumab, the patient developed urticarial wheals appearing on the abdomen, trunk, and head and neck. This eruption resolved after a 1-week course of prednisone $40 \mathrm{mg}$ and fexofenadine. In the patient's first MM biomarker assessment after the start of ixekizumab, drops in her kappa:lambda ratio, kappa free light chain, and M-protein levels were observed (Fig. 1). A further decrease in these clinical markers was found in a second assessment 2 weeks later. Thereafter, these markers fluctuated in a range below the highest peak. The patient reported minimal efficacy of ixekizumab for her psoriasis and thus decided to discontinue use after 2 months.

\section{Case Series Using Digital Crowdsourcing}

To further understand clinical features and treatment responses among patients with both MM and psoriasis, health data crowdsourced on HealthTree were used. A total of 1505 HealthTree enrollees had verified physician diagnoses of myeloma, and psoriasis was present in $4.9 \%$ $(n=73)$ of this population. Surprisingly, psoriasis was one of the most frequent co-morbidities found in the HealthTree myeloma patient population, alongside conditions such as osteopenia, inflammatory bowel disease, and gout. Our questionnaire (Table S1) was administered to HealthTree patients $(n=73)$ with verified health records showing diagnosis of 


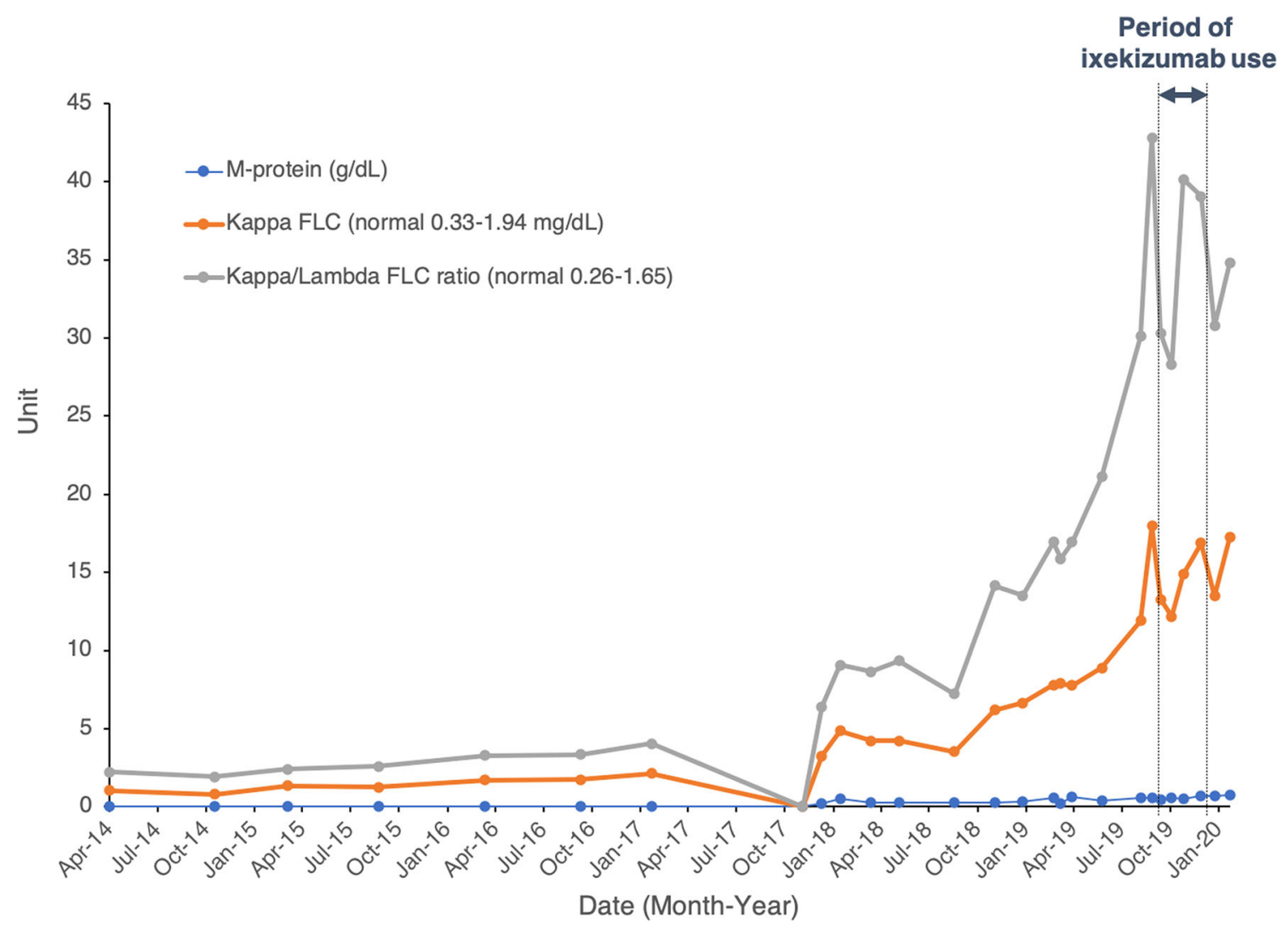

Fig. 1 Myeloma markers for Patient 1. Standard myeloma prognostic laboratory tests, including $\mathrm{M}$-protein, kappa free light chain (FLC), and kappa/lambda FLC ratio were tracked across the ixekizumab treatment start and end

both psoriasis and MM. The questionnaire yielded 18 complete responses, including from Patient 1 described above. Findings pertaining to psoriasis are summarized in Table 1 , while myeloma features are summarized in Table 2 . Characteristics evaluated in the questionnaire include gender, current age, age of diagnosis, psoriasis type, psoriasis treatment history, myeloma type and genetic features, and myeloma association with bone damage, hypercalcemia, or osteopenia.

Among respondents, the mean age of psoriasis diagnosis was 35.9 (SD 8.2), while the mean age of myeloma diagnosis was 57.1 (SD 8.6). In most patients, diagnosis of psoriasis occurred well before diagnosis of myeloma (mean difference of 21.2 years). Seven out of 18 respondents identified as female (38.9\%), and the most common type of psoriasis diagnosed was plaque date. The double arrows indicate the period of ixekizumab treatment from September 2019 to December 2019

(44.4\%), followed by palm/sole (16.7\%), guttate $(11.1 \%)$, and inverse (5.6\%). Two patients $(11.1 \%)$ indicated that their psoriasis treatment (topical steroids) had improved their MM, and $33.3 \%$ indicated they were uncertain. No patients reported that psoriasis treatment had negatively influenced myeloma progression, and $50 \%$ were uncertain. Psoriasis treatments were most commonly topical steroids, followed by tar, phototherapy, heliotherapy, and antifungal or immunosuppressive agents.

Myeloma diagnoses included MM (72.2\%), smoldering myeloma (33.3\%), and monoclonal gammopathy of undetermined significance (27.8\%) - several patients were diagnosed with multiple subtypes. The types of myeloma represented included IgG kappa (50.0\%), IgA kappa (11.1\%), IgA lambda (11.1\%), and IgM lambda $(5.6 \%)$ as well as $22.2 \%$ uncertain. Among 


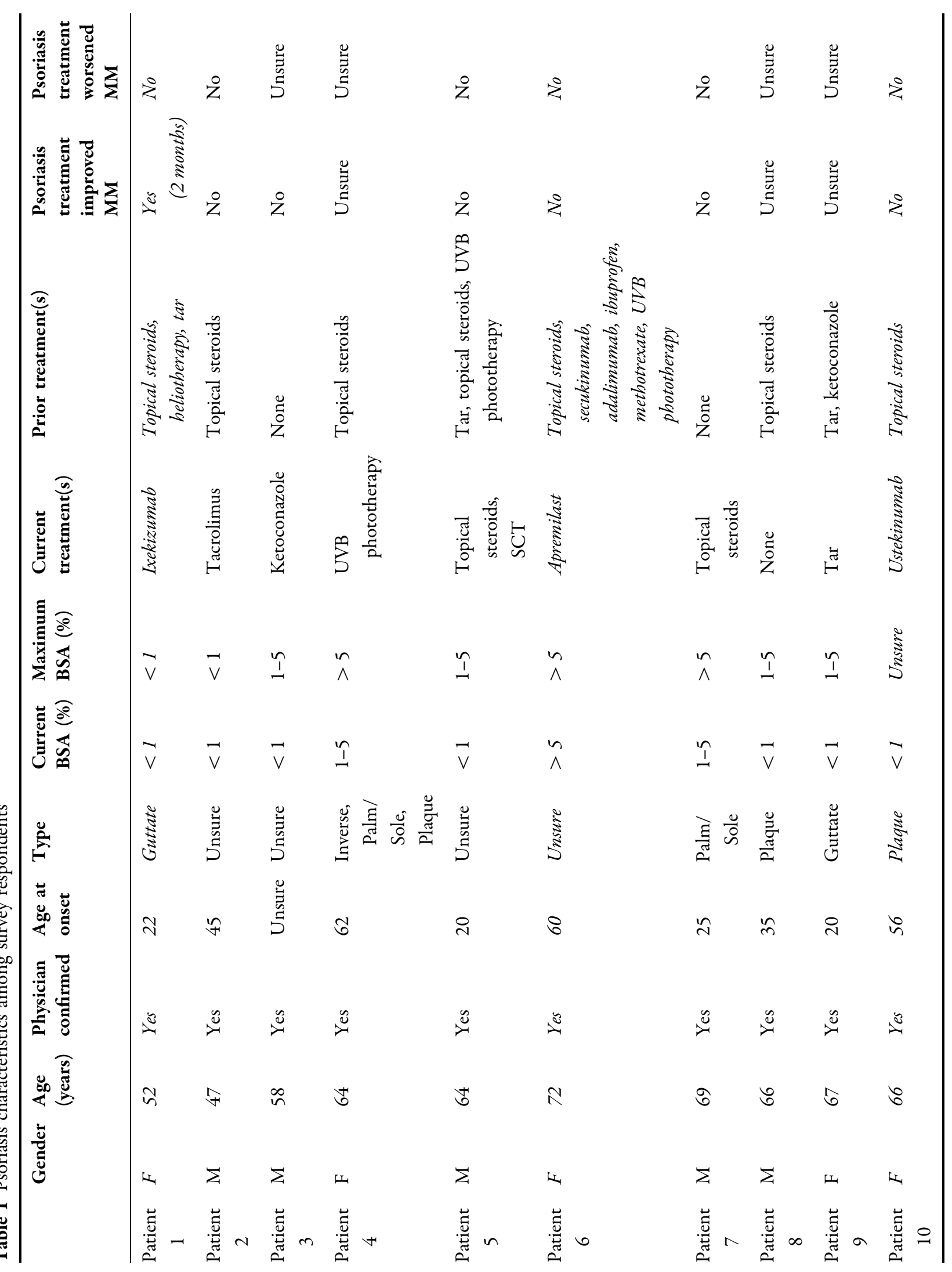


820

Dermatol Ther (Heidelb) (2020) 10:815-827

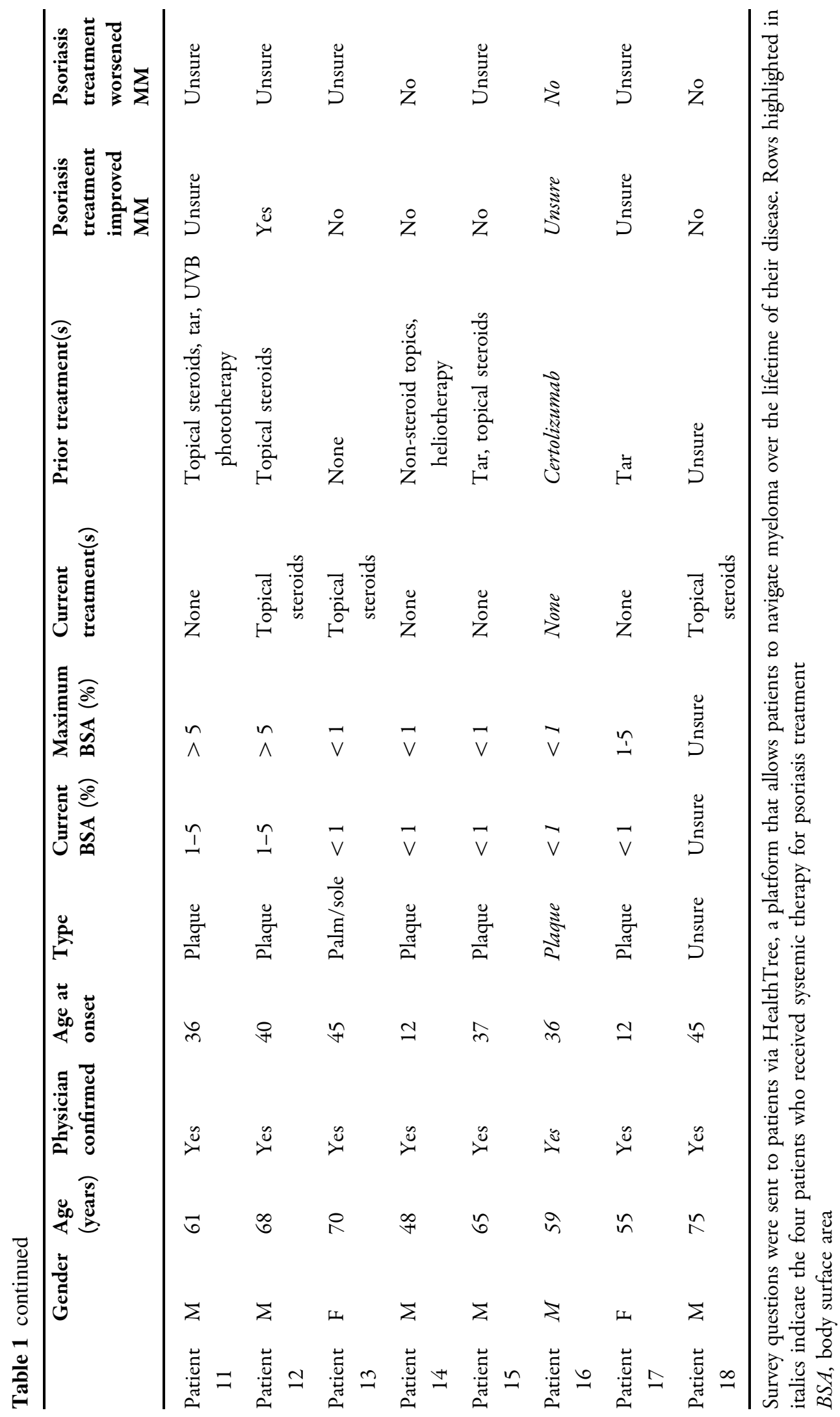

么 Adis 


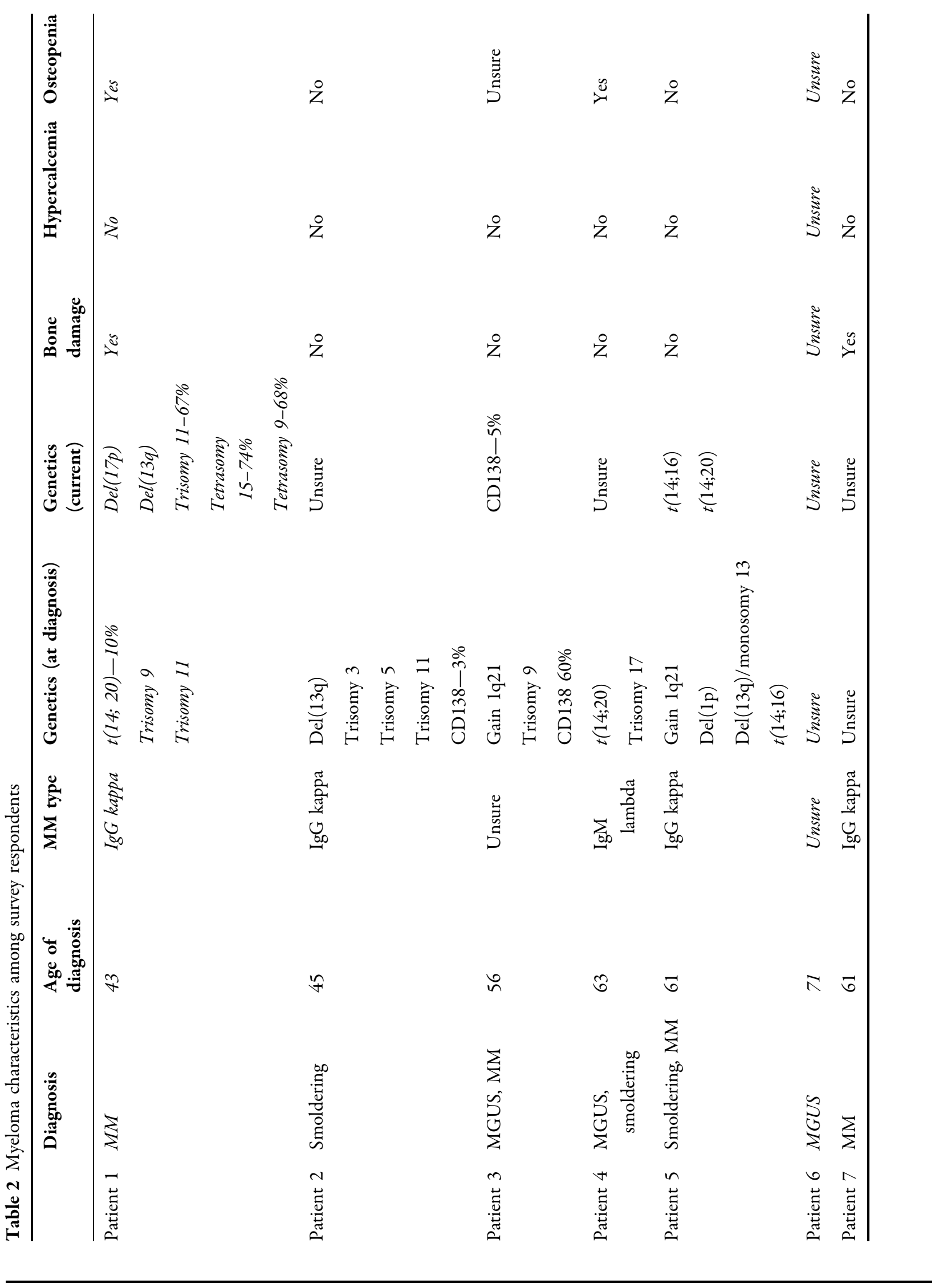




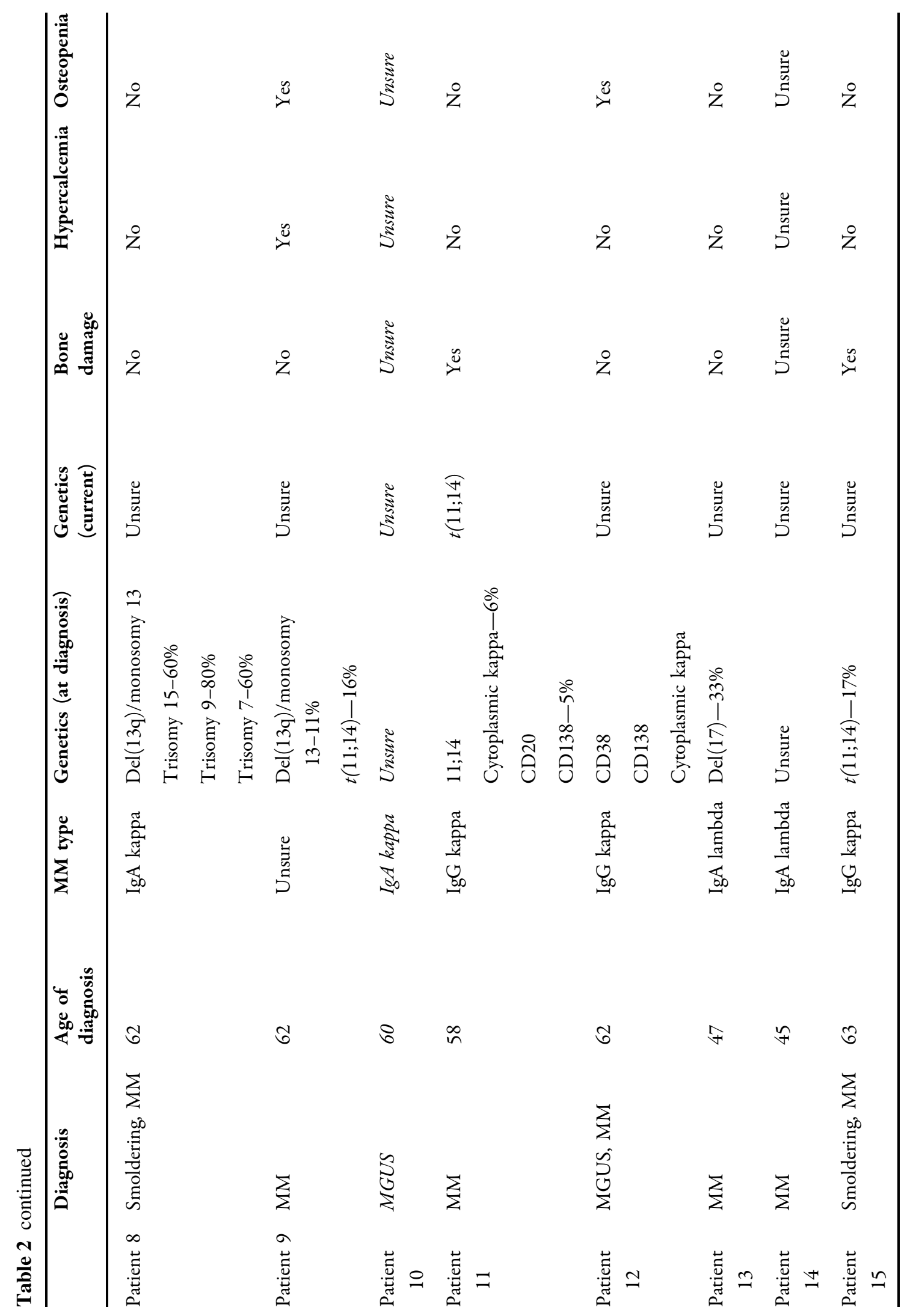




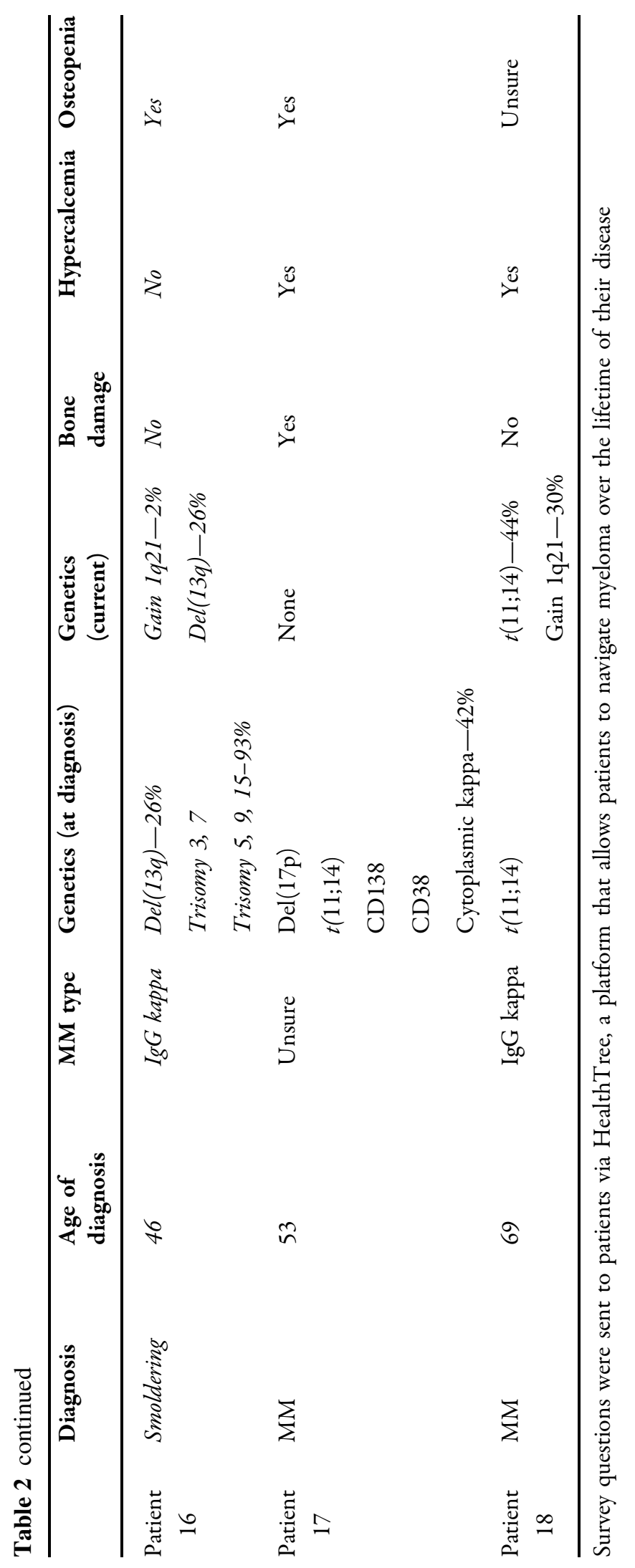


respondents, $29.4 \%$ reported bone damage (e.g., fracture), $16.7 \%$ reported hypercalcemia, and $33.3 \%$ reported osteopenia.

Out of all respondents, Patients 1, 6, 10, and 16 had received treatment with systemic therapies: ixekizumab (anti-IL17), apremilast (antiPDE4), ustekinumab (anti-IL12/IL23), and certolizumab (anti-TNF), respectively. These four patients affirmed that systemic psoriasis treatment did not worsen their initial MM, with two patients demonstrating temporary or unclear improvement in MM control following systemic therapy. No other differences specific to this subgroup of patients were found.

Consent for publication was received from the index patient; the digitally crowdsourced patient data were determined to be exempt by the Western Institutional Review Board (IRB) under exemption code 45 CFR § 46.104(d)(2). This case report was conducted according to the principles of good clinical practice guidelines ICH GCP and according to the ethical principles of the Declaration of Helsinki.

\section{DISCUSSION AND CONCLUSIONS}

To our knowledge, no studies have examined the clinical characteristics and treatment of patients diagnosed with both psoriasis and myeloma, prompting our examination of this subpopulation using HealthTree. MM is a hematologic cancer of terminally differentiated plasma cells with an increasing global burden, accounting for approximately $10 \%$ of all hematologic malignancies in the US in 2017 [7]. Patients present with a distinct set of symptoms indicating monoclonal gammopathy as the cause of end-organ damage, including hypercalcemia, renal insufficiency, anemia, and bone disease with lytic lesions. In combination with these clinical features, a diagnosis is made basedon blood and urine laboratory tests, imaging, and bone marrow aspiration or biopsy. While smoldering myeloma and monoclonal gammopathy of undetermined significance (MGUS) often precede MM, treatment is not typically initiated until disease progresses to MM, a more symptomatic state. Following diagnostic workup and risk stratification, treatment strategy typically involves chemotherapy followed by autologous stem cell transplantation or immunomodulatory maintenance to prevent future $\mathrm{MM}$ recurrence. Patients are followed with prognostic markers including M-protein, bone marrow biopsy, and kappa and lambda free light chain (FLC) concentrations to assess for myeloma progression.

Among respondents who did know their myeloma genetic diagnosis, only four subtypes-IgG kappa, IgA kappa, IgA lambda, and IgM lambda-out of ten possible subtypes were represented. The majority of respondents were IgG kappa, which suggests that future research is needed to determine whether patients with certain myeloma subtypes may have a predisposition toward the development of psoriasis, or vice versa.

Our case series gives important insights for patients with a dual diagnosis of psoriasis and MM. Epidemiologic studies have shown that psoriatic patients have elevated risks for the incidence of certain cancers such as lymphoma, possibly due to chronic inflammatory processes [8]. Regarding MM, a study of $>890,000$ psoriasis patients and 4.4 million age- and sex-matched controls showed an elevated incidence of $\mathrm{MM}$ in psoriasis patients (HR 1.26 [95\% CI 1.07-1.48]) [9]. Moreover, the use of certain systemic immunotherapies for the management of psoriasis may increase the risk of cancer. For example, use of anti TNF- $\alpha$ biologics has been associated with increased non-melanoma skin cancer rates, particularly for cutaneous squamous cell carcinoma [10].

Several immunomodulators and biologic therapies for psoriasis currently exist, including but not limited to adalimumab, etanercept, infliximab, certolizumab, tildrakizumab, guselkumab, risankizumab, ustekinumab, brodalumab, ixekizumab, and secukinumab. While the literature has not reported on the use of biologic therapies for the specific management of psoriasis in patients affected by MM, the use of these biologics has been studied in MM patients with other diseases of immune etiology, including Crohn's disease and rheumatic arthritis. In two case studies of MM patients with Crohn's disease, neither adalimumab nor infliximab worsened MM symptom severity, 
while etanercept used for rheumatic arthritis was non-significantly associated with reduced risk of MM in a retrospective case-control study [11-13]. No other studies examined the use of biologics for psoriasis in patients with MM, pointing to the need for additional research on immunomodulatory biologics in patients with psoriasis and MM.

Our index patient with psoriasis and MM was treated with ixekizumab, an IL-17 inhibitor. IL17 is a known molecular driver of psoriasis, and recent preclinical research has also implicated the involvement of IL-17 in MM disease progression [14, 15]. For instance, anti-IL-17A human monoclonal antibody (mAb) administration has demonstrated the ability to reduce MM proliferation, growth, and progressive bone damage in mice [15]. In this study, Prabhala et al. demonstrated that anti-IL-17A mAb downregulates MM cell growth by blocking IL-6 production and significantly reducing osteoclast proliferation. Immune-competent mouse studies showed a significant reduction in tumor volume following subcutaneous mAb injection. Earlier studies further elucidated the role of IL17 production by T-helper $17\left(\mathrm{~T}_{\mathrm{H}} 17\right)$ cells in promoting $\mathrm{MM}$ cell growth and contributing to general immune dysfunction [14]. IL-17 leads to myeloma cell colony formation, adhesion to bone marrow stromal cells, and significantly increased growth in vivo in murine xenograft models using MM patient tissue. Interestingly, a recent publication by Duffield et al. indicated that in classical Hodgkin lymphoma the IL-17/ IL-23 axis is also key to the ongoing pathogenesis, which is resistant to checkpoint inhibition by existing anti-PD-1 drugs [16]. Therefore, further study of anti-IL-17 biologic use in psoriasis patients with $\mathrm{MM}$, and potentially other hematologic malignancies, is warranted. Our first patient demonstrated temporary improvement in her MM biomarkers after starting ixekizumab, but discontinued ixekizumab after 2 months because of lack of psoriasis efficacy and unclear effect of ixekizumab on her MM biomarkers in the long term. It will be interesting to determine whether IL-17 blockade is beneficial in blocking the progression of these malignancies and whether such therapy can be safely used in the setting of psoriasis or other
anti-IL-17 approved conditions such as psoriatic arthritis and ankylosing spondylitis.

HealthTree is a powerful tool that enables the clinical study of health data from myeloma patients who are also diagnosed with other conditions, including psoriasis. This case series is an example of how crowdsourcing can facilitate the easy collection of deidentified clinical data of patients across the globe to enable research and analysis for accelerated treatment findings and practices. For instance, the prevalence of psoriasis and myeloma is between $0.91 \%$ and $3.15 \%$ in the US, respectively, and the co-occurrence would have been even rarer, but HealthTree allowed us to quickly reach our target demographic for clinical study [17]. This includes the gathering of valuable clinical characteristics-for instance, our study reports that patients generally develop psoriasis much earlier than MM with an average difference of 21.2 years in time of diagnosis, which has implications for close monitoring of psoriasis therapies given new malignant diagnoses.

Our survey results show that among the four patients who had been treated with systemic immunotherapies for psoriasis, none reported the worsening of myeloma during the study period. Encouragingly, two patients indicated a temporary (Patient 1) or unclear (Patient 16) improvement in $\mathrm{MM}$ progression as indicated by the prognostic markers described above (Mprotein, kappa FLC, and kappa to lambda FLC ratios). Specifically, Patient 1 was treated with ixekizumab, an IL-17 inhibitor, and demonstrated a temporary improvement in her myeloma prognostic markers for 2 months before reverting back to her original trajectory. Therefore, our results warrant further investigation of the use of systemic immunotherapies such as ixekizumab in treating patients with psoriasis and MM.

In conclusion, the analysis of crowdsourced health data through platforms such as HealthTree can yield significant results for treatment of dermatologic conditions such as psoriasis. This case series is the first to examine the clinical characteristics and treatment of patients diagnosed with both psoriasis and MM. Encouraging results from four patients in this study indicate that systemic biologic 
immunotherapies for psoriasis can potentially be used in individuals with comorbid malignancies such as MM. Further research in this area, particularly the use of anti-IL-17 biologics, should be explored.

\section{ACKNOWLEDGEMENTS}

We thank the participants of the study.

Funding. No funding or sponsorship was received for this study or publication of this article. Wilson Liao is funded in part by grants from the National Institutes of Health (U01AI119125) and has served as a research investigator for Abbvie, Amgen, Janssen, Novartis, Pfizer, Regeneron, Sanofi, and TRex Bio. Jenny M. Ahlstrom has served as a patient advisor for BMS, Amgen, Takeda, and Janssen. Myeloma Crowd has received funding for HealthTree from Takeda, Sanofi, and GSK.

Authorship. All named authors meet the International Committee of Medical Journal Editors (ICMJE) criteria for authorship for this article, take responsibility for the integrity of the work as a whole, and have given their approval for this version to be published.

Disclosures. Joy Q. Jin, Jenny M. Ahlstrom, Nathan W. Sweeney have nothing to disclose. Wilson Liao is a member of the journal's Editorial Board.

Compliance with Ethics Guidelines. Consent for publication was received from the index patient; the digitally crowdsourced patient data were determined to be exempt by the Western Institutional Review Board (IRB) under exemption code 45 CFR $\S 46.104(d)(2)$. This case report was conducted according to the principles of good clinical practice guidelines ICH GCP and according to the ethical principles of the Declaration of Helsinki.

Open Access. This article is licensed under a Creative Commons Attribution-NonCommercial 4.0 International License, which permits any non-commercial use, sharing, adaptation, distribution and reproduction in any medium or format, as long as you give appropriate credit to the original author(s) and the source, provide a link to the Creative Commons licence, and indicate if changes were made. The images or other third party material in this article are included in the article's Creative Commons licence, unless indicated otherwise in a credit line to the material. If material is not included in the article's Creative Commons licence and your intended use is not permitted by statutory regulation or exceeds the permitted use, you will need to obtain permission directly from the copyright holder. To view a copy of this licence, visit http://creativecommons.org/licenses/by$\mathrm{nc} / 4.0 /$.

\section{REFERENCES}

1. Sanchez IM, Shankle L, Wan MT, Afifi L, Wu JJ, Doris $\mathrm{F}$, et al. Building a citizen pscientist: advancing patient-centered psoriasis research by empowering patients as contributors and analysts. Dermatol Ther [Internet]. 2018;8(3):405-23.

2. The Myeloma Crowd Launches Psoriasis/Multiple Myeloma Study Using Its HealthTree ${ }^{\circledR}$ Platform [Internet]. [cited 2020 May 18]. https://www. prnewswire.com/news-releases/the-myelomacrowd-launches-psoriasis-multiple-myeloma-studyusing-its-healthtree-platform-300929387.html. Accessed 18 May 2020.

3. Blauvelt A, Chiricozzi A. The immunologic role of IL-17 in psoriasis and psoriatic arthritis pathogenesis. Clin Rev Allergy Immunol [Internet]. 2018;55(3):379-90.

4. Rajkumar SV. Multiple myeloma: 2020 update on diagnosis, risk-stratification and management. Am J Hematol [Internet]. 2020;95(5):548-67.

5. Dommasch E, Gelfand JM. Is there truly a risk of lymphoma from biologic therapies? Dermatol Ther [Internet]. 2009;22(5):418-30.

6. Papp KA, Leonardi C, Menter A, Ortonne JP, Krueger JG, Kricorian G, et al. Brodalumab, an anti-interleukin-17-receptor antibody for psoriasis. N Engl J Med [Internet]. 2012;366(13):1181-9.

7. Kumar SK, Rajkumar V, Kyle RA, van Duin M, Sonneveld P, Mateos MV, et al. Multiple myeloma. Nat Rev Dis Prim [Internet]. 2017;3:17046. 
8. Chiesa Fuxench ZC, Shin DB, Ogdie Beatty A, Gelfand JM. The risk of cancer in patients with psoriasis: a population-based cohort study in the health improvement network. JAMA Dermatol [Internet]. 2016;152(3):282-90.

9. Lee JH, Kim HJ, Han KD, Kim HN, Park YM, Lee JY, et al. Cancer risk in 892089 patients with psoriasis in Korea: a nationwide population-based cohort study. J Dermatol [Internet]. 2019;46(2):95-102.

10. Asgari MM, Ray GT, Geier JL, Quesenberry CP. Malignancy rates in a large cohort of patients with systemically treated psoriasis in a managed care population. J Am Acad Dermatol [Internet]. 2017;76(4):632-8.

11. Park SY, Kim JM, Kang HJ, Kim M, Han JJ, Maeng $\mathrm{CH}$, et al. Crohn's disease and smoldering multiple myeloma: a case report and literature review. Intest Res. 2017;15(2):249-54.

12. Mielke F, Schweigert M. Safe adalimumab therapy for rheumatoid arthritis in a patient with pre-existing multiple myeloma. Nat Clin Pract Rheumatol. 2008;4(4):218-21.
13. Calip GS, Patel PR, Sweiss K, Wu Z, Zhou J, Asfaw AA, et al. Targets of biologic disease-modifying antirheumatic drugs and risk of multiple myeloma. Int J Cancer. 2020. https://doi.org/10.1002/ijc.32891.

14. Prabhala RH, Pelluru D, Fulciniti M, Prabhala HK, Nanjappa P, Song W, et al. Elevated IL-17 produced by TH17 cells promotes myeloma cell growth and inhibits immune function in multiple myeloma. Blood [Internet]. 2010;115(26):5385-92.

15. Prabhala RH, Fulciniti $M$, Pelluru D, Rashid N, Nigroiu A, Nanjappa P, et al. Targeting IL-17A in multiple myeloma: a potential novel therapeutic approach in myeloma. Leukemia [Internet]. 2016;30(2):379-89.

16. Duffield AS, Ascierto ML, Anders RA, Taube JM, Meeker AK, Chen S, et al. Th17 immune microenvironment in Epstein-Barr virus-negative Hodgkin lymphoma: implications for immunotherapy. Blood Adv [Internet]. 2017;1(17):1324-34.

17. Rachakonda TD, Schupp CW, Armstrong AW. Psoriasis prevalence among adults in the United States. J Am Acad Dermatol [Internet]. 2014;70(3):512-6. 